\title{
Service-Oriented Collaboration Model Based on Active Rule for Sensor Networks
}

\author{
Jinhui Chen \\ School of Computer \& Software, Nanjing University of Information Science \& Technology, Nanjing, \\ 210044, China \\ cjh@nuist.edu.cn
}

Keywords: Sensor networks; Service; Collaboration; Active rule

\begin{abstract}
This paper presented an approach for modeling and implementing the architecture of sensor networks collaborative service using active rule model. Considering service framework and description model which were necessary for sensor network application, an active rule model was defined by integrating service framework with event based collaborative service process model. The analysis indicated that the service process model provided the related solutions from two aspects of configurability and expansibility for sensor networks collaboration. The results imply that active rule can easily be constructed, while ensuring good reconfigurability.
\end{abstract}

\section{Introduction}

Sensor system is a system that integrates a number of sensors. Sensor and sensor system are unified as sensor resource. Sensor network consists of a number of distributed sensor resources that communicate with each other. Since multiple sensors can supply more information in time and space, sensor network technique has been widely used. Due to the existence of a large number of sensor protocols and interfaces, many applications integrate sensor resources through private mechanism rather than through collaborative layer. Private mechanisms do not facilitate the sharing and interoperability of sensor resource. Therefore, we need to build a service-oriented sensor network that serves as a bridge between sensor resources and applications. Service-oriented sensor network is an infrastructure that can use sensor resources in standard ways, such as discovery, access, event and warning[1,2]. Sensor network coordination is a method for collaborative observation, information fusion, and online service of sensors in a distributed network environment. By collaboration, we concentrate sensor resources and services as an organic whole, and establish links between them[3,4]. In the face of a variety of sensor resources, services, and a wide range of complex applications of sensor networks, collaboration solution of sensor networks focuses on the following problems: how to easily get multi-source sensor data and provide better sensor network services.

Demirbas et al. propose that Twitter can provide an "open" publish-subscribe infrastructure for sensors and smartphones, and pave the way for ubiquitous crowd-sourced sensing and collaboration applications[5]. Mori et al. propose a support environment for wireless sensor networks applications development, and design a language to describe high-level specification of cooperative applications on wireless sensor networks[6]. Kar et al. consider a power-constrained sensor network, consisting of multiple sensor nodes and a fusion center, and propose an optimal collaborative strategy by minimizing the cumulative transmission power subject to a maximum distortion constraint[7]. Fanaei et al. investigate the problem of linear spatial collaboration for distributed estimation in wireless sensor networks. The main novelty is the derivation of an optimal power-allocation scheme[8]. Rossi et al. deal with spectrum sensing for cognitive radio scenarios where the decision fusion center exploits array processing, explore the impact of user cooperation and orthogonal transmissions among secondary users on the reporting channel[9]. Santos et al. present a decentralized algorithm for detecting damage in structures by using a wireless sensor and actuator network, and make use of cooperative information fusion for calculating a damage coefficient[10].

The above researches effectively improve the efficiency of development for sensor networks and 
running quality, but a key problem of sensor networks collaboration for further study and implementation is how to provide high level abstraction in order to solve problem of multi-sensor information perception, fusion and collaboration. This paper proposes a collaboration model for designing and developing service-oriented sensor networks application. Our model supports the following features:

(1) An integrated service framework based on active rule for sensor networks.

(2) Event based collaborative service process model, which is based on event distribution mechanism, takes active service execution based on rule as a back-end operating environment.

(3) Service implementation from the two aspects of configurability and expansibility.

\section{Service Framework}

Sensor network should provide the following basic services, such as sensor planning service, web notification service, sensor observation service, sensor event service and sensor alert service. Through sensor planning service, clients can determine the feasibility of collecting data from one or more sensors, and submit requests for either data collection or configuration processing. Through web notification service, clients can perform synchronous or asynchronous operations between multiple services. Through sensor observation service, clients can get a description of a sensor platform. Sensor event service provides a series of subscriptions to manage events.

In the service oriented architecture of sensor networks, service providers provide data and publish services, service requesters is application objects oriented to sensor network service. Fig. 1 shows a service framework based on active rule for sensor networks, which is divided into four layers, namely sensor networks layer, primitive rule processing layer, composite rule processing layer and application layer.

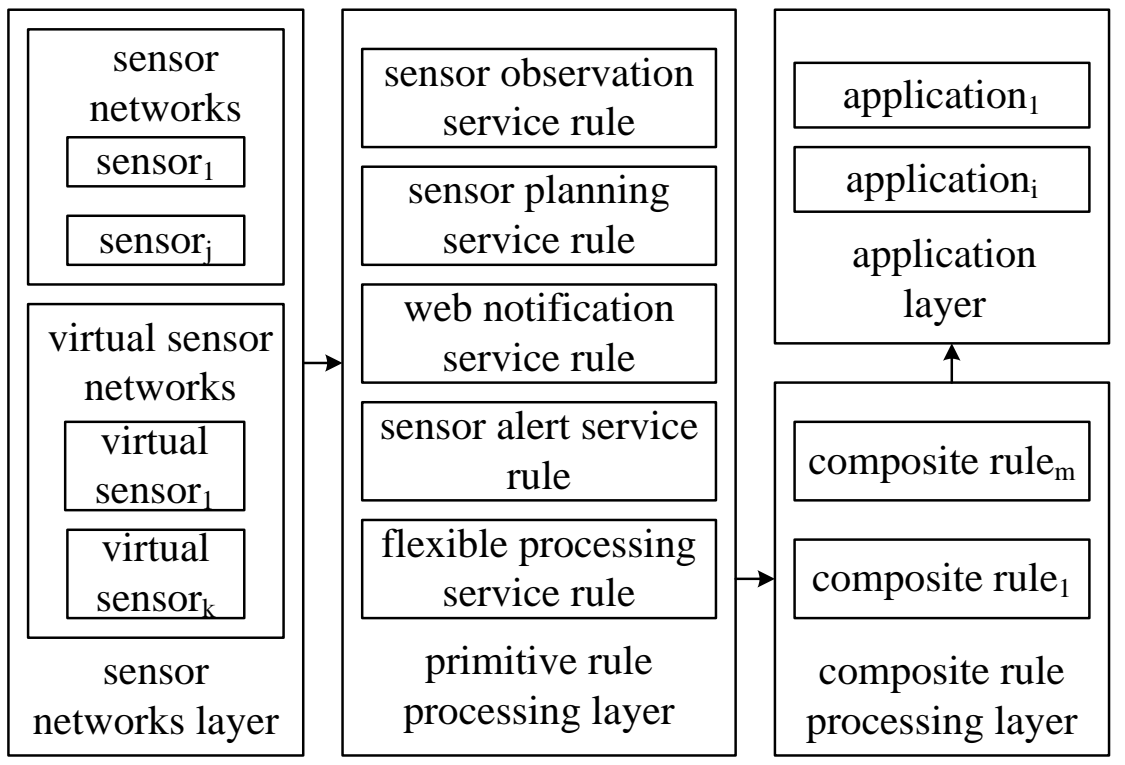

Figure 1. Service framework based on active rule for sensor networks

The sensor networks layer provides the measured data for sensor networks service, which comprises both entity sensors and non-entity sensors. Non-entity sensors can provide data to enrich the scope of sensor, to expand the range of applications and to help the unified integration of multi-source observations. Service layer provides rule-based sensor networks service. From the perspective of service ability, service is divided into primitive service and composite service, where composite service is the integration of primitive services, or the integration of primitive services and composite services. As client, the application layer includes users and terminal devices. This layer is equivalent to the service providers and the registration center in the service oriented architecture. If a service 
provider has registered a service, a service requester can discover the service through the registration center.

\section{Service Process Model}

Service Process. The service collaboration model based on active rule mainly includes the following elements: service process, activity, event and rule. Service process refers to collaborative service process that is across organizational boundaries, and can be composed of multiple activities, events and rules. Each service process can include a number of sub-processes. Activity is the basic element of service process. In the practice of running a service process, the activity implementation depends on the service implementation supported by different organizations. Event reflects the state changes of service or environment related to specific service process in service process environment. Event is the entity information that drives service process, and can be described by notification. Rule represents the logical relationship between events and activities. When an event that satisfies a specific condition is triggered, it activates the corresponding rule and executes the corresponding activity. A rule can be represented by a triple, and denoted as $R=\{\mathrm{Ev}, \mathrm{Ac}, \mathrm{Tr}\}$, where $\mathrm{Ev}$ is a set of events, Ac is a set of activities, and $\mathrm{Tr}$ represents event-to-activity migration or activity-to-event migration.

The service collaboration model has two characteristics. First, each service process contains a number of services, the services are called while the process in being executed. Moreover, the coordination model of service process is is based on event-driven rule. Second, the model realizes the separation of event and rule. Event is responsible for describing the context information of the service process, and rule is used as a trigger mechanism for event-based service, so as to meet the loose-coupled requirement in dynamic and changeable service environment.

Event Detection. When sensor sends information to the upper layer application in the form of simple event, event processing system first needs to integrate the rich semantic information to generate composite events, then complex event is submitted to rule processing system to make decision, and finally service process performs the corresponding operations and completes the required tasks. Sensor networks event processing is an advanced form of complex query, compared with the traditional event processing, it has the following characteristics:

(1) Situation relevance. In order to identify event, we need to take into account the specific conditions and background of event, including the occurrence of the event space, time and objects and other related information.

(2) Relationship between cause and effect. In sensor networks, events are usually dependent on each other, a single event may have no actual meaning. In event space, the causal order can reflect the logical relationship between separate events. By using the time sequence of event, we can establish the link between the global state and the local state, so as to reflect the causal relationship between events.

In order to detect complex event, an event detection system first describes the structure and semantics of complex event by event description language, that is to define the corresponding active rules, then the rules are transformed into the corresponding composite event detection model. When processing event stream, event detection system can filter primitive events according to constraint conditions of the composite event detection model. When all the constraints in a composite event are met, the composite event is successfully detected.

Executive Process of Service. In the executive process of service, the internal and external events of service process trigger the corresponding rules, then the rules perform actions defined in service process, and finally during the course of performing the activities, new events will be published. Therefore, there is an active relationship between activity, event and rule in service process.

The specific structure of active rule is as follows: $R=\{\operatorname{Ev}($ SituationValue, EventProfile), $\operatorname{Tr}$ (SituationValueExpression), Ac\}. Where the parameter SituationValue defines the event structure of a specific scenario, its parameters are the attribute values and attribute types of event. By using logical connectors, the parameter EventProfile defines a complex event schema that is composed of a series of primitive events. These two parameters SituationValue and EventProfile illustrate a scenario event and 
its composition. The parameter SituationValueExpression shows the constraint conditions that SituationValue needs to meet.

In order to identify rule, we need to translate a specific rule into an automaton model. The model depends on the sequence of events and various constraints in rule, and consists of one or more specific event sequences. At the run time of an automaton, different event notifications may result in the following results.

(1) When an event notification meets the initialization conditions of an automaton, an instance of the automaton is created, and then is migrated to the initial state.

(2) The automaton is transferred from the state $S_{i}$ to the state $S_{i+1}$.

(3) When an event triggers an automaton to migrate to the final state, this means that an event schema has been correctly identified; or when an instantiation of an automaton becomes invalid, so the farther processing is not needed. In both the cases, the instantiation of the automaton is deleted.

\section{Service Implementation}

Sensor network adopt a service oriented architecture, and service description language exposes services. Clients can use these service information provided by service description language to interact with services. The service module achieves all kinds of operations specified by service standards. Realization technology of sensor network service involves the following questions: storage constraints of database, multi source database adapter and multi sensor adapter. For the problem of storage constraints, we establish the mapping relation between XML Schema and relational database. According to elements and their relationships, we store sensor network services, and establish links between elements and queries. For the adapter problems of both multi source database and multi sensor, we use abstract factory method to realize multi object matching. That is, sensor network service database is abstracted into an abstract class, each specific database is used as an instance.

The service implementation architecture includes a unified service description language and the implementations of services, where the unified service description language is used to expose the descriptions, bindings, interface types, and operations of service. In the architecture, there are two kinds of interaction forms, namely, the interaction between client and service, and the interaction between service and sensor. The interaction process between client and service is as follows: through sensor observation service, client first gets the service's capability information and observation data, then calls sensor planning service, and finally, when sensor planning service completes task or when sensor observation service can provide data, web notification service and sensor event service notify client. The interaction process between service and sensor is as follows. First, sensor planning service directly submits service to sensor. Secondly, sensor observation service interacts with sensor. The interactions can be divided into two categories: active and passive. Active interaction refers to the process, in which sensor dynamically inserts observation data into sensor observation service by the operations provided by sensor observation service. Passive interaction refers to the process: when sensor observation service can not provide data service, the service is triggered to subscribe to data from sensor and virtual sensor. Finally, when subscription data is ready, the data is inserted into sensor observation service, at the same time, the notification service is triggered in order to notify user.

\section{Conclusions}

In this paper, we propose an integrated service framework based on active rule for sensor networks. The framework is defined by an event based collaborative service process model, which is based on event distribution mechanism. The service process model takes active service execution based on rule as a back-end operating environment, and provides the related solutions from two aspects of configurability and expansibility. The results imply that active rule can easily be constructed, while ensuring good reconfigurability. 


\section{Acknowledgements}

This work was sponsored by open fund project of Jiangsu provincial research and development center of intelligent sensor network engineering technology, China.

\section{References}

[1] A.Ostmark, J.Eliasson, P.Lindgren, et al. An Infrastructure for Service Oriented Sensor Networks[J]. Journal of Computers, 2006, 1(5).

[2] N.Mohamed, J.Aljaroodi. A survey on service-oriented middleware for wireless sensor networks[C]. Service Oriented Computing and Applications, 2011.

[3] R.K.Sharma, D.B.Rawat. Advances on Security Threats and Countermeasures for Cognitive Radio Networks: A Survey[J]. IEEE Communications Surveys and Tutorials, 2015, 17(2): 1023-1043.

[4] H.Salarian, K.Chin, F.Naghdy, et al. Coordination in wireless sensor-actuator networks: A survey[J]. Journal of Parallel and Distributed Computing, 2012, 72(7): 856-867.

[5] M.Demirbas, M.A.Bayir, C.G.Akcora, et al. Crowd-sourced sensing and collaboration using twitter[C]. World of Wireless, Mobile and Multimedia Networks, 2010.

[6] S.Mori, T.Umedu, A.Hiromori, et al. Data-centric programming environment for cooperative applications in WSN[C]. Integrated Network Management, 2013.

[7] S.Kar, P.K.Varshney. Linear Coherent Estimation With Spatial Collaboration[J]. IEEE Transactions on Information Theory, 2013, 59(6): 3532-3553.

[8] M.Fanaei, M.C.Valenti, A.Jamalipour, et al. Optimal Power Allocation for Distributed BLUE Estimation with Linear Spatial Collaboration[C]. International Conference on Acoustics, Speech, and Signal Processing, 2014.

[9] P.S.Rossi, D.Ciuonzo, G.Romano, et al. Orthogonality and Cooperation in Collaborative Spectrum Sensing through MIMO Decision Fusion[J]. IEEE Transactions on Wireless Communications, 2013, 12(11): 5826-5836.

[10] I.L.Santos, L.Pirmez, L.F.Carmo, et al. A Decentralized Damage Detection System for Wireless Sensor and Actuator Networks[J]. IEEE Transactions on Computers, 2016, 65(5): 1363-1376. 